\title{
Kink-Soliton, Singular-Kink-Soliton and Singular- Periodic Solutions for a New Two-Mode Version of the Burger-Huxley Model: Applications in Nerve Fibers and Liquid Crystals
}

\author{
Marwan Alquran \\ Jordan University of Science and Technology \\ Tukur Abdulkadir Sulaiman \\ Biruni Universitesi \\ Abdullahi Yusuf ( $\nabla$ yusufabdullahi@fud.edu.ng) \\ Federal University Dutse, Nigeria https://orcid.org/0000-0002-8308-7943
}

\section{Research Article}

Keywords: Kink, Singular kink, Singular periodic, Burger-Huxley model, Simpli-fied bilinear method, Polynomial-function method, Kudryashov-expansion method

Posted Date: March 31st, 2021

DOl: https://doi.org/10.21203/rs.3.rs-351353/v1

License: (c) (i) This work is licensed under a Creative Commons Attribution 4.0 International License.

Read Full License

Version of Record: A version of this preprint was published at Optical and Quantum Electronics on April 22nd, 2021. See the published version at https://doi.org/10.1007/s11082-021-02883-2. 


\title{
Kink-soliton, singular-kink-soliton and singular-periodic solutions for a new two-mode version of the Burger-Huxley model: Applications in nerve fibers and liquid crystals
}

\author{
Marwan Alquran ${ }^{1}$, Tukur Abdulkadir Sulaiman ${ }^{2,3}$, Abdullahi Yusuf ${ }^{2,3, *}$ \\ marwan04@just.edu.jo, sulaiman.tukur@fud.edu.ng, yusufabdullahi@fud.edu.ng \\ ${ }^{1}$ Department of Mathematics and Statistics, Jordan University of Science \& Technology, Irbid 22110, Jordan \\ ${ }^{2}$ Department of Computer Engineering, Biruni University, Istanbul, Turkey \\ ${ }^{3}$ Department of Mathematics, Federal University Dutse, Jigawa, Nigeria \\ ${ }^{*}$ Corresponding author
}

\section{Abstract}

New two-mode version of Burger-Huxley equation is derived using Korsunsky's operators. The new model arises in the applications of nerve fibers and liquid crystals and describes the interaction of two symmetric waves moving simultaneously in the same direction. Kink-soliton, singular-kink-soliton and singular-periodic solutions are obtained to this model by means of the simplified bilinear method, polynomial-function method and the Kudryashov-expansion method. A comprehensive graphical analysis is conducted to show the physical aspects of this new type of nonlinear equations. Finally, all obtained solution are verified by direct substitution in the model.

Key Words: Kink; Singular kink; Singular periodic; Burger-Huxley model; Simplified bilinear method; Polynomial-function method; Kudryashov-expansion method.

Mathematics Subject Classification: 26A33, 35F25, 35C10.

\section{Introduction}

The generalized Burger-Huxley equation is a nonlinear partial differential equation of first order in time $t$, and reads

$$
\phi_{t}-\phi_{x x}-\alpha \phi \phi_{x}-\beta \phi(1-\phi)(\phi-\gamma)=0
$$


where $\phi=\phi(x, t)$ and $\alpha, \beta, \gamma$ are general real scalars. For the case of $\alpha=0$ and $\gamma=1$, (1.1) is reduced to the Huxley model which represents the propagation of nerve pulses in nerve fibers and wall motion in liquid crystals [1]. For $\beta=0$ and $\gamma=1,(1.1)$ is the Burgers' equation which describes the field of wave propagation in nonlinear dissipative models. The Burger-Huxley (1.1), describes the interaction of convection terms against diffusion transmission [1]. The $\left(G^{\prime} / G\right)$-expansion method [2] and the auxiliary Riccati equation method [3] are used to solve (1.1) and hyperbolic function solutions, trigonometric function solutions and rational solutions are obtained. In [4], The Cole-Hopf transformation, that transforms the Burgers equation into the heat-conduction equation, is used to obtain exact solutions of the Burgers-Huxley. Finally, the tanh-coth method [5] and the tanh-expansion method [6] are used to solve the Burger-Huxley where solutions of type kinks and periodic-waves are obtained.

The main goal of this work is to extend the Burger-Huxley equation into a second order in time and to explore its solutions by using different schemes. The new version of the Burger-Huxley is called two-mode Burger-Huxley (TMBH) and to be derived based on Korsunsky-Wazwaz transformations. To do so, we consider the following mappings

$$
\begin{aligned}
\phi_{t} & \rightarrow \phi_{t t}-s^{2} \phi_{x x} \\
\alpha \phi \phi_{x}+\beta \phi(1-\phi)(\phi-\gamma) & \rightarrow\left(\frac{\partial}{\partial t}-a s \frac{\partial}{\partial t}\right)\left\{\alpha \phi \phi_{x}+\beta \phi(1-\phi)(\phi-\gamma)\right\}, \\
\phi_{x x} & \rightarrow\left(\frac{\partial}{\partial t}-a s \frac{\partial}{\partial t}\right)\left\{\phi_{x x}\right\} .
\end{aligned}
$$

The parameter $s$ is regarded as the phase-velocity. The parameters $a$ and $b$ are the nonlinearity and dispersive factors and their values are dominated absolutely by 1 . Therefore, the TMBH takes the following form

$$
\phi_{t t}-s^{2} \phi_{x x}-\left(\frac{\partial}{\partial t}-a s \frac{\partial}{\partial t}\right)\left\{\alpha \phi \phi_{x}+\beta \phi(1-\phi)(\phi-\gamma)\right\}-\left(\frac{\partial}{\partial t}-a s \frac{\partial}{\partial t}\right)\left\{\phi_{x x}\right\}=0 .
$$

In (1.3), if $s=0$ and integrating the resulting equation once with respect to $t$, we get the standard single-mode Burger-Huxley equation (1.1). In this regard, many two-mode equations are established using Korsunsky's sense, and some solitary waves solutions are reported by different ansatze methods, see $[9,10,11,12,13,14,15]$ and $[16,17,18,19,20]$.

The contribution of this work is to explore the TMBH, as a new member of the family of two-mode equations, and to find possible solitary solutions by means of the simplified bilinear method, polynomial-function method and the Kudryashov-expansion method. 


\section{Simplified bilinear method}

In this section, we use the simplified bilinear method [21, 22, 23] to find the one-soliton solution to (1.3). This technique requires the following auxiliary functions:

$$
\begin{aligned}
& h(x, t)=\mu x-c t, \\
& v(x, t)=e^{h(x, t)}, \\
& u(x, t)=1+\lambda v(x, t), \\
& \phi(x, t)=A(\ln (u(x, t)))_{x} .
\end{aligned}
$$

Substitution of (2.5) in the linear terms of (1.3), and solving for the speed $c$, we get

$$
c=\frac{1}{2}\left(\beta \gamma-\mu^{2} \pm \sqrt{\left.\left(\mu^{2}-\beta \gamma\right)^{2}+4 \mu s(a \beta \gamma+\mu(s-b \mu))\right)}\right) .
$$

Note that in (2.8), the wave transform $\zeta=\mu x-c t$ has two value of $c$, "two speeds". Then, we insert (2.7) in (1.3) and solve the resulting equation for the unknowns $A$ and $\mu$ to reach at the following outputs:

$$
\begin{aligned}
& a=b, \\
& A=\frac{-\alpha \mp \sqrt{\alpha^{2}+8 \beta}}{2 \beta}, \\
& \mu=\frac{\alpha \pm \sqrt{\alpha^{2}+8 \beta}}{4} .
\end{aligned}
$$

Therefore, the one-soliton solution to the TMBH is

$$
\phi(x, t)=\frac{\lambda e^{\mu x-c t}}{1+\lambda e^{\mu x-c t}} .
$$

It is worth to mention that for $\lambda>0$, the solution (2.10) is of type kink. While as, $\lambda<0$ gives singular-kink. Now, as the speed $c$ has two distinct values (2.8), the dynamic concept of the soliton solutions given in (2.10) that it propagates as two-waves moving simultaneously in the same direction and their interaction depends on the increase of the embedded phase-velocity $s$, see Figure 1.

\section{Polynomial-function method}

The goal of this section is to further explore more new solutions to the TMBH by using another scheme. We consider the polynomial-function method [29, 30]. First, we transform (1.3) through the wave transform $\zeta=\mu(x-c t)$ into the following simplified differential equation:

$$
\mu^{2}\left(c^{2}-s^{2}\right) \phi^{\prime}+\mu(c+a s)\left(\alpha \mu \phi \phi^{\prime}+\beta \phi(1-\phi)(\phi-\gamma)\right)+\mu^{3}(c+b s) \phi^{\prime \prime}=0,
$$

where $\phi=\phi(\zeta)$. The suggested solution to (3.11) is

$$
\phi(\zeta)=b_{0}+b_{1} Y+b_{2} Y^{-1},
$$



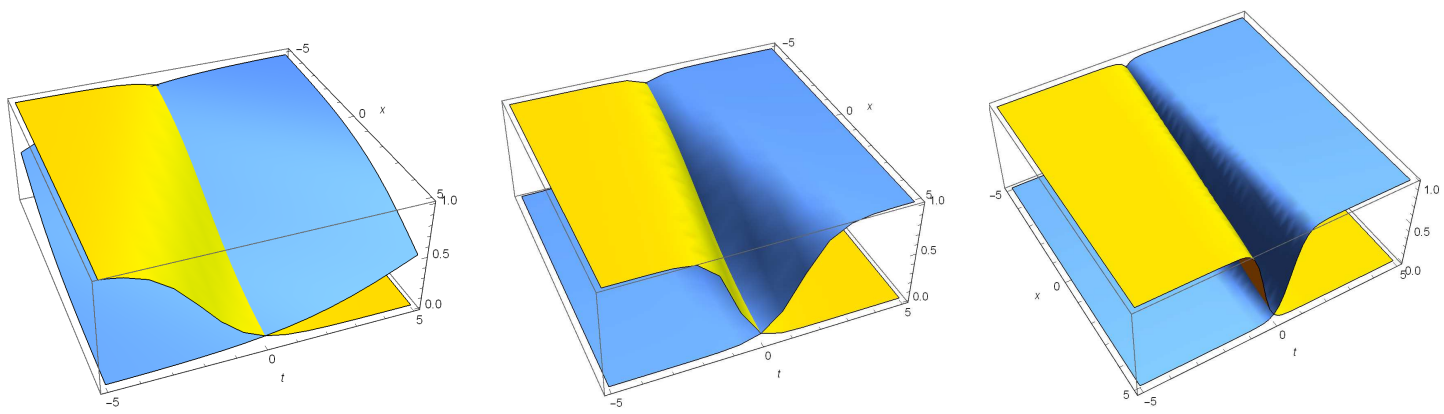

Figure 1: 3D interaction of moving left-right waves upon increasing the phase velocity $s=$ 2, 5, 10. $\alpha=\beta=\gamma=1, b=0.5, \lambda=1$. Simplified bilinear method.

where the variable $Y=Y(\zeta)$ satisfies the auxiliary differential equation

$$
Y^{\prime}=Y^{2}+A Y+B
$$

For this stage, we need to find the required higher derivatives of $\phi(\zeta)$, based on the relationship given in (3.13). Implicit differentiation of (3.12) gives

$$
\begin{aligned}
\phi^{\prime}(\zeta) & =(Y(A+Y)+B)\left(b_{1}-\frac{b_{2}}{Y^{2}}\right) \\
\phi^{\prime \prime}(\zeta) & =\frac{(Y(A+Y)+B)\left(b_{1} Y^{3}(A+2 Y)+b_{2}(A Y+2 B)\right)}{Y^{3}} .
\end{aligned}
$$

Substitution of (3.12) and (3.14) in (3.11), results in a finite series in terms of negative and positive powers of $Y$. Setting each coefficient of $Y^{i}$ to zero leads to a non-algebraic system in the unknowns $b_{0}, b_{1}, b_{2}, \mu$ and $c$. Now, we solve the obtained system based on the states of the free constants $A$ and $B$ defined in (3.13). We consider the following two cases:

Case I: $A=0, \quad B \neq 0$, leads to the following outputs:

$$
\begin{aligned}
a & =b \\
b_{0} & =0, \quad b_{1}=-\frac{\sqrt{\gamma}}{\sqrt{B}}, \quad b_{2}=0 \\
\mu & =\frac{\sqrt{\gamma}\left(\alpha-\sqrt{\alpha^{2}+8 \beta}\right)}{4 \sqrt{B}}, \\
c & =\frac{-\left(\gamma^{2} \sqrt{\alpha^{2}+8 \beta}+\gamma \sqrt{\alpha^{2}+8 \beta}+\alpha(\gamma+1) \gamma\right)}{4 \gamma} \\
\mp & \frac{\sqrt{2} \sqrt{\gamma^{2}\left((\gamma+1)^{2}\left(\alpha \sqrt{\alpha^{2}+8 \beta}+\alpha^{2}+4 \beta\right)-4 b(\gamma+1) s\left(\sqrt{\alpha^{2}+8 \beta}+\alpha\right)+8 s^{2}\right)}}{4 \gamma} .
\end{aligned}
$$


But, the solution of (3.13) is $Y=Y(\zeta)=\sqrt{B} \tan (\sqrt{B} \zeta)$. Accordingly, the solution of TMBH is

$$
\phi(x, t)=\sqrt{\gamma} \tan \left(\frac{1}{4} \sqrt{\gamma}\left(\alpha-\sqrt{\alpha^{2}+8 \beta}\right)(x-c t)\right)
$$

Case II: $A \neq 0, \quad B=0$, leads to:

$$
\begin{aligned}
a & =b \\
b_{0} & =0, \quad b_{1}=-\frac{\gamma}{A}, \quad b_{2}=0 \\
\mu & =\frac{\gamma\left(\sqrt{\alpha^{2}+8 \beta}+\alpha\right)}{4 A}, \\
c & =-\frac{\gamma \sqrt{\alpha^{2}+8 \beta}-2 \sqrt{\alpha^{2}+8 \beta}+\alpha \gamma+2 \alpha}{8} \\
& \pm \frac{\sqrt{\alpha\left(\gamma^{2}-4\right) \Omega+\alpha^{2}\left(\gamma^{2}+4\right)+4 \beta(\gamma-2)^{2}-8 b s((\gamma-2) \Omega+\alpha(\gamma+2))+32 s^{2}}}{\sqrt{32}}
\end{aligned}
$$

where $\Omega=\sqrt{\alpha^{2}+8 \beta}$. The solution of (3.13) is $Y=\frac{A}{d e^{-A \mu(x-c t)}-1}$. Accordingly, the solution of TMBH is

$$
\phi(x, t)=\frac{\gamma}{d e^{-\frac{1}{4} \gamma\left(\sqrt{\alpha^{2}+8 \beta}+\alpha\right)(x-c t)}-1} .
$$

The solution given in (3.18) is of type kink-soliton when $(d<0)$ and of type singular-kink for $(d>0)$. On the other side, it is clear that the solution given in (3.16) is of type singularperiodic and the obtained left-wave and right-wave solutions are presented in Figure 2. The interaction of these two periodic solutions upon increasing the phase velocity $s$ is shown in Figure 3.
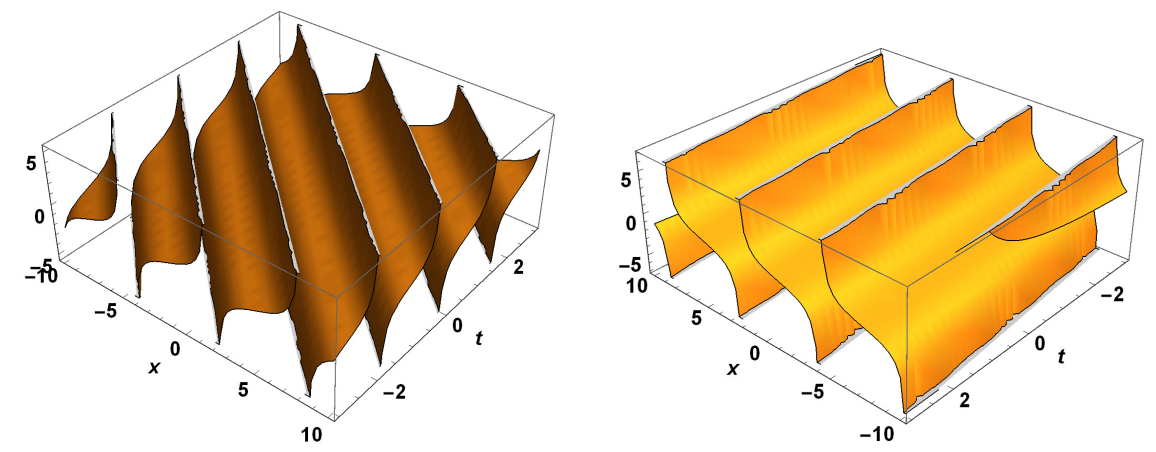

Figure 2: The Left-wave and the right-wave solutions of TMBH as depicted in (3.16). $\alpha=$ $\beta=\gamma=1, s=1$. 

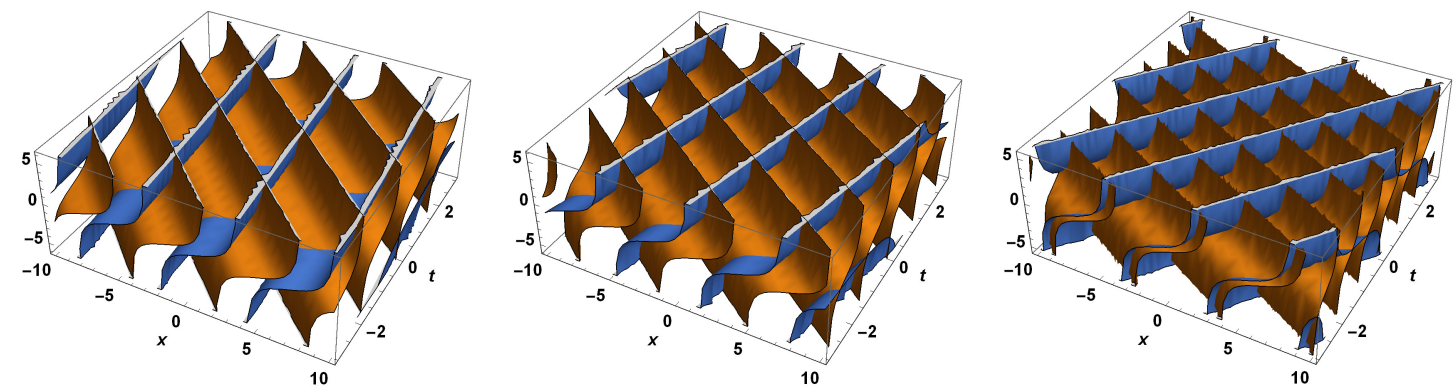

Figure 3: The interaction of the two periodic solutions upon increasing the phase velocity $s=1,3,5$ to the TMCH. $\alpha=\beta=\gamma=1$. Polynomial-function method.

\section{Kudryashov-expansion method}

Another goal of this work is to present the Kudryashov-expansion method [24, 25, 26, 27, 28] to seek other physical-type solutions that the TMBH could have. By the wave transform $\eta=x-c t$, the TMBH is reduced to the following simplified differential equation

$$
\left(c^{2}-s^{2}\right) \phi^{\prime}+(c+a s)\left(\alpha \phi \phi^{\prime}+\beta \phi(1-\phi)(\phi-\gamma)\right)+(c+b s) \phi^{\prime \prime}=0,
$$

where $\phi=\phi(\eta)$. The suggested solution to (4.19) is

$$
\phi(\zeta)=\lambda_{0}+\lambda_{1} Y
$$

where the variable $Y=Y(\eta)$ satisfies the auxiliary differential equation

$$
Y^{\prime}=\mu Y(Y-1)
$$

Differentiating (4.20) based on (4.21), we get

$$
\begin{aligned}
\phi^{\prime}(\zeta) & =\lambda_{1} \mu Y(Y-1) \\
\phi^{\prime \prime}(\zeta) & =\lambda_{1} \mu^{2} Y(Y-1)(2 Y-1)
\end{aligned}
$$

Now, we insert (4.20) and (4.22) in (4.19), to obtain a polynomial of degree 3 in the variable $Y$ whose coefficients are

$Y^{0}=\beta\left(\lambda_{0}-1\right) \lambda_{0}\left(\gamma-\lambda_{0}\right)(a s+c)$,

$Y^{1}=-\lambda_{0}(a s+c)(2 \beta(\gamma+1)-\alpha \mu)+3 \beta \lambda_{0}^{2}(a s+c)+a \beta \gamma s-\mu s(b \mu+s)+c^{2} \mu+c\left(\beta \gamma-\mu^{2}\right)$,

$Y^{2}=\lambda_{1}(a s+c)(-\alpha \mu+\beta \gamma+\beta)+\lambda_{0}(a s+c)\left(\alpha \mu-3 \beta \lambda_{1}\right)-\mu\left(3 b \mu s-c^{2}+3 c \mu+s^{2}\right)$,

$Y^{3}=\alpha \lambda_{1} \mu(a s+c)-\beta \lambda_{1}^{2}(a s+c)+2 \mu^{2}(b s+c)$.

Since the above coefficients are identical to zeros, the first coefficient is zero if

$$
\lambda_{0}=\gamma
$$


Accordingly, setting the other coefficients to zeros, produce the following results:

$$
\begin{aligned}
a & =b, \\
\gamma & =1, \\
\lambda_{1} & =\frac{\mu\left(\alpha \mp \sqrt{\alpha^{2}+8 \beta}\right)}{2 \beta}, \\
\mu & =\frac{1}{4}\left(\sqrt{\alpha^{2}+8 \beta}+\alpha\right), \\
c & =\frac{1}{8}\left(\Delta-3 \alpha \mp \sqrt{-6 \alpha \Delta+10 \alpha^{2}+16 b s \Delta-48 \alpha b s+8 \beta+64 s^{2}}\right), \\
\Delta & =\sqrt{\alpha^{2}+8 \beta} .
\end{aligned}
$$

Therefore, the two-wave solutions of TMBH is

$$
\phi(x, t)=\frac{1}{d e^{-\frac{1}{128}(\Delta+\alpha)^{2}\left(t\left(\Delta-3 \alpha \mp \sqrt{2} \sqrt{-3 \alpha \Delta+5 \alpha^{2}+8 b s(\Delta-3 \alpha)+4 \beta+32 s^{2}}\right)-8 x\right)}-1}+1 .
$$

We should point here that the sign of the free parameter in (4.26) determine different physical shapes for the TMBH. For example, if $d>0$, the resulting type is kink-soliton which is the same finding obtained in Section 2. For $d>0$, it is of singular-kink type. Figure 4 shows the the so-called left-wave and the right-wave and their interaction. Figure 5, presents the impact of the phase velocity $s$ acting on the overlapping of these two moving left-right waves.
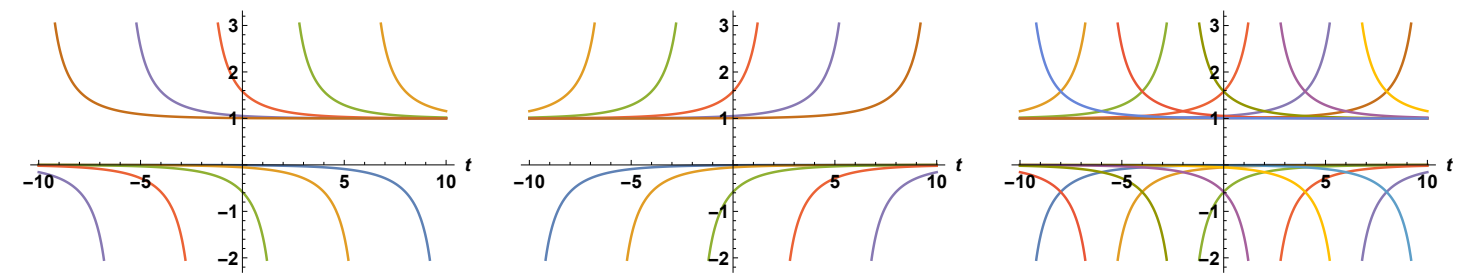

Figure 4: Profile solutions of the left, right and left-right waves to the TMBH as depicted in (4.26). $\alpha=\beta=1, s=0.5, b=0.5, d=1$.
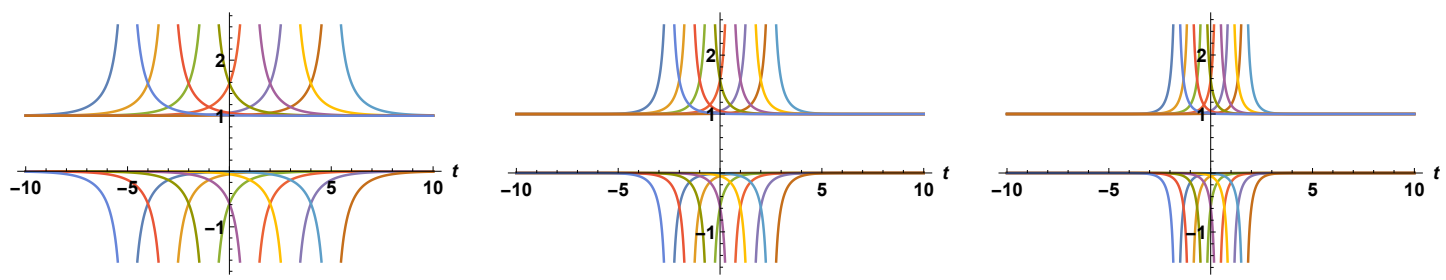

Figure 5: 2D interaction of moving left-right waves upon increasing the phase velocity $s=$ 1, 2, 3. $\alpha=\beta=1, b=0.5, d=1$. Kudryashov-expansion method. 


\section{Discussion and concluding remarks}

The Burger-Huxley equation od second order in time is presented in this work for the first time. This model has been introduced under the name of two-mode Burger-Huxley TMBH. Solitary-wave solutions with different physical-structures are obtained to TMBH. The following items are some validations and insights regarding the obtained solutions of the new model:

- The obtained two-waves of TMBH are always symmetric and their interaction id affected by increasing their phase velocity.

- If $s=0$, the TMBH is reduced to the single $\mathrm{BH}$. To validate this fact, consider $s=0$ in (2.8), then $c=\beta \gamma-\mu^{2}$ which is a single wave speed and thus a single wave-solution. Same observations can be verified for $c$ given in (3.15), (3.17) and (4.25).

- The obtained solution of TMBH given in (3.16) is acting like wall motion in liquid crystals, see Figure 2.

- Nerve pulses can propagate in nerve fibers through two different routes and maintain its physical shape.

\section{References}

[1] Wang X.Y., Zhu Z.S., Lu Y.K., Solitary wave solutions of the generalized BurgersHuxley equation. J. Phys. A: Math. Gen. 23 (1990) 271-274.

[2] Manafian J., Lakestani M., Solitary wave and periodic wave solutions for Burgers, Fisher, Huxley and combined forms of these equations by the $\left(G^{\prime} / G\right)$-expansion method. PRAMANA-Journal of physics 85(1) (2015) 31-52.

[3] Wang G.W., Liu X.Q., Zhang Y.Y., New explicit solutions of the generalized BurgersHuxley equation. Vietnam J Math (2013) 41: 161-166.

[4] Yefimova O.Y., Kudryashov N.A., Exact solutions of the Burgers-Huxley equation. Journal of Applied Mathematics and Mechanics 68(3) (2004) 413-420.

[5] Wazwaz A.M., Analytic study on Burgers, Fisher, Huxley equations and combined forms of these equations. Applied Mathematics and Computation 195 (2008) 754-761.

[6] Wazwaz A.M., Travelling wave solutions of generalized forms of Burgers, Burgers-KdV and Burgers-Huxley equations. Applied Mathematics and Computation 169 (2005) 639656.

[7] Korsunsky S.V., Soliton solutions for a second-order KdV equation. Phys. Lett. A 185 (1994) 174-176.

[8] Wazwaz A.M., Two-mode fifth-order KdV equations: necessary conditions for multiplesoliton solutions to exist. Nonlinear Dynamics 87(3) (2017) 1685-1691. 
[9] Lee C.T., Multi-soliton solutions of the two-mode KdV. PhD Thesis, Oxford University, Oxford (2007).

[10] Alquran M., Jarrah A., Jacobi elliptic function solutions for a two-mode KdV equation. Journal of King Saud University-Science 31(4) (2019) 485-489.

[11] Yassin O., Alquran M., Constructing new solutions for some types of two-mode nonlinear equations. Applied Mathematics and Information Sciences 12(2) (2018) 361-367.

[12] Alquran M., Yassin O., Dynamism of two-mode's parameters on the field function for third-order dispersive Fisher: application for fibre optics. Optical and Quantum Electronics 50(9) (2018): 354.

[13] Wazwaz A.M., Two-mode Sharma-Tasso-Olver equation and two-mode fourth-order Burgers equation: Multiple kink solutions. Alexandria Eng. J. 57(3) (2018) 1971-1976.

[14] Wazwaz A.M., A study on a two-wave mode Kadomtsev-Petviashvili equation: conditions for multiple soliton solutions to exist. Mathematical Methods in the Applied Sciences 40(11) (2017) 4128-4133.

[15] Wazwaz A.M., Two wave mode higher-order modified KdV equations: Essential conditions for multiple soliton solutions to exist. International Journal of Numerical Methods for Heat \& Fluid Flow 27(10) (2017) 2223-2230.

[16] Jaradat A., Noorani MSM., Alquran A., Jaradat H.M., Construction and solitary wave solutions of two-mode higher-order Boussinesq-Burger system. Advances in Difference Equations (2017) 2017: 376.

[17] Jaradat H.M., Alquran A., Syam I., A reliable study of new nonlinear equation: Two-mode Kuramoto-Sivashinsky. International Journal of Applied and Computational Mathematics 4(2) (2018): 64.

[18] Jaradat I., Alquran M., Ali M., A numerical study on weak-dissipative two-mode perturbed Burgers' and Ostrovsky models: right-left moving waves. Eur. Phys. J. Plus (2018) 133: 164 .

[19] Jaradat I., Alquran M., Momani S., Biswas A., Dark and singular optical solutions with dual-mode nonlinear Schrodinger's equation and Kerr-law nonlinearity. Optik 172 (2018) 822-825.

[20] Alquran M., Jaradat I., Multiplicative of dual-waves generated upon increasing the phase velocity parameter embedded in dual-mode Schrodinger with nonlinearity Kerr laws. Nonlinear Dynamics 96 (2019) 115-121.

[21] Syam M., Jaradat H.M., Alquran M., A study on the two-mode coupled modified Korteweg-de Vries using the simplified bilinear and the trigonometric-function methods. Nonlinear Dynamics 90(2) (2017) 1363-1371. 
[22] Jaradat H.M., Syam M., Alquran M., A two-mode coupled Korteweg-de Vries: multiplesoliton solutions and other exact solutions. Nonlinear Dynamics 90(1) (2017) 371-377.

[23] Alquran M., Jaradat H.M., Syam M., A modified approach for a reliable study of new nonlinear equation: two-mode Korteweg-de Vries-Burgers equation. Nonlinear Dynamics 91(3) (2018) 1619-1626.

[24] Alquran M., Jaradat I., Baleanu D., Shapes and dynamics of dual-mode Hirota-Satsuma coupled KdV equations: Exact traveling wave solutions and analysis. Chinese Journal of Physics 58 (2019) 49-56.

[25] Abu Irwaq I., Alquran M., Jaradat I., New dual-mode Kadomtsev-Petviashvili model with strong-weak surface tension: analysis and application. Advances in Difference Equations 2018: 433 (2018).

[26] Alquran M., Jaradat I., Ali M., Baleanu D., The dynamics of new dual-mode Kawahara equation: Interaction of dual-waves solutions and graphical analysis. Physica Scripta 95 (2020) 045216.

[27] Jaradat I., Alquran M., Construction of solitary two-wave solutions for a new two-mode version of the Zakharov-Kuznetsov equation. Mathematics 8(7) (2020) 1127.

[28] Alquran M., Jaradat I., Ali M., Al-Ali N., Momani S., Development of spreading symmetric two-waves motion for a family of two-mode nonlinear equations. Heliyon 6(6) (2020) e04057.

[29] Huang W.H., A polynomial expansion method and its application in the coupled Zakharov-Kuznetsov equations. Chaos Solitons and Fractals 29(2) (2006) 365-371.

[30] Deng G.F., Gao Y.T., Integrability, solitons, periodic and travelling waves of a generalized $(3+1)$-dimensional variable-coefficient nonlinear-wave equation in liquid with gas bubbles. Eur. Phys. J. Plus (2017) 132: 255. 


\section{Figures}
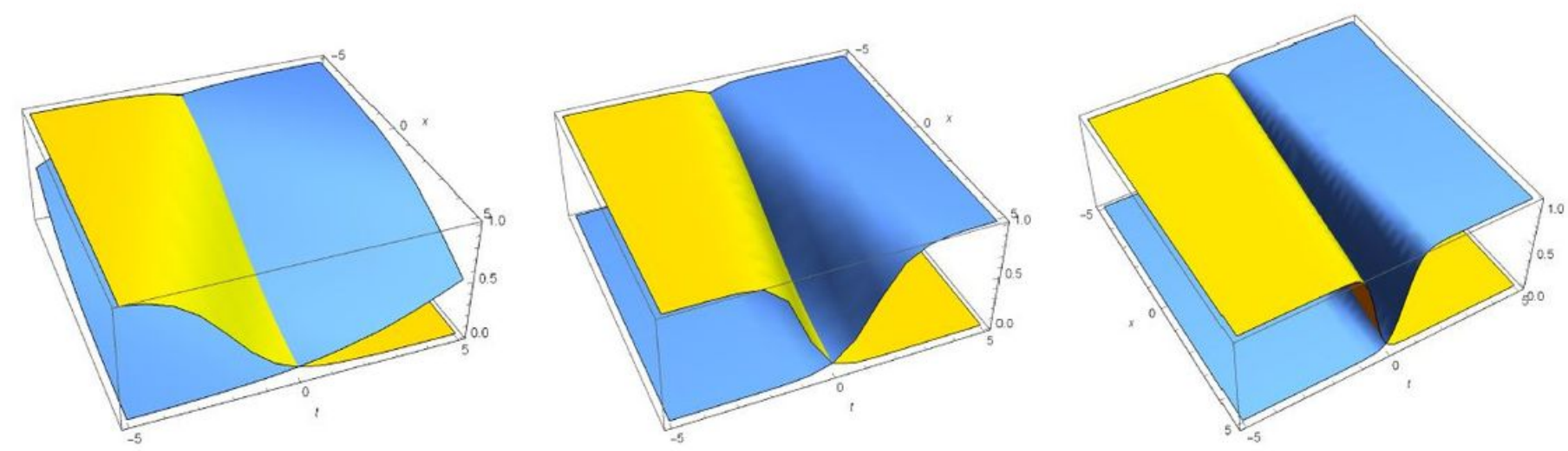

Figure 1

3D interaction of moving left-right waves upon increasing the phase velocity $s=2 ; 5 ; 10 . a=\beta=y=1 ; b=$ $0: 5 ; \lambda=1$. Simplied bilinear method.
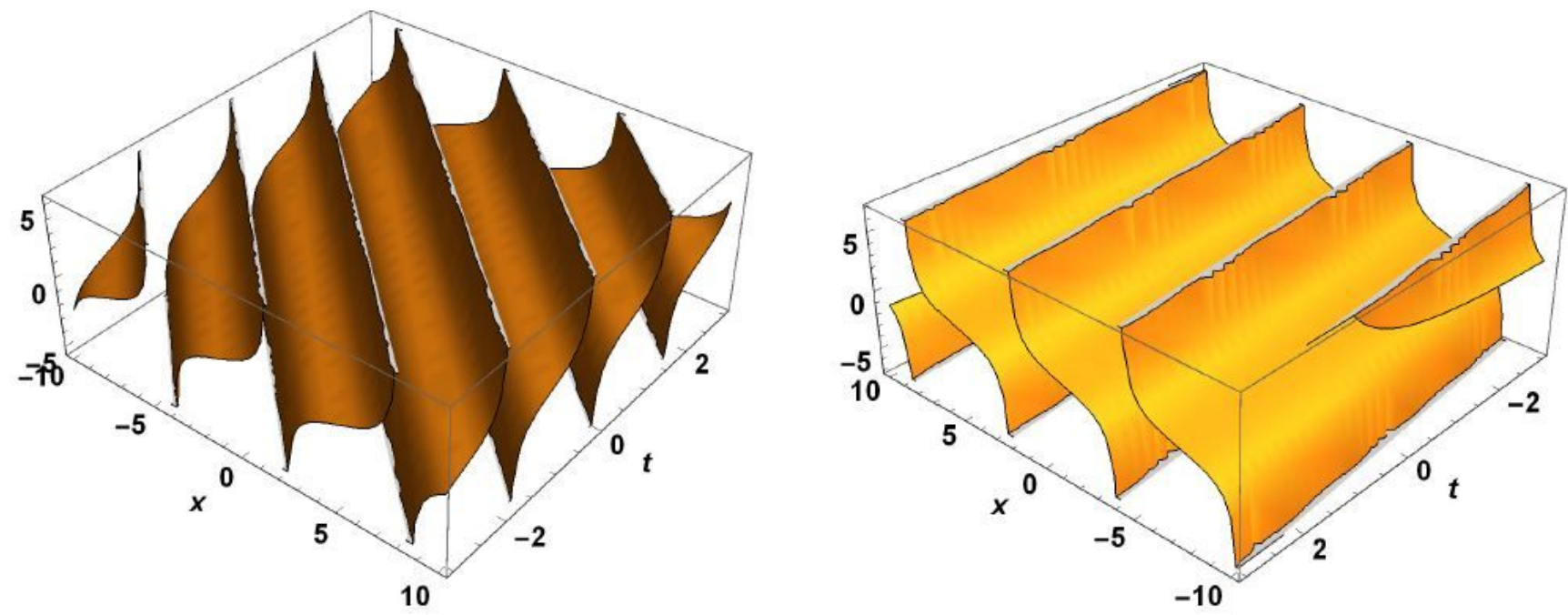

Figure 2

The Left-wave and the right-wave solutions of TMBH as depicted in (3.16). $\alpha=\beta=\gamma=1 ; s=1$.
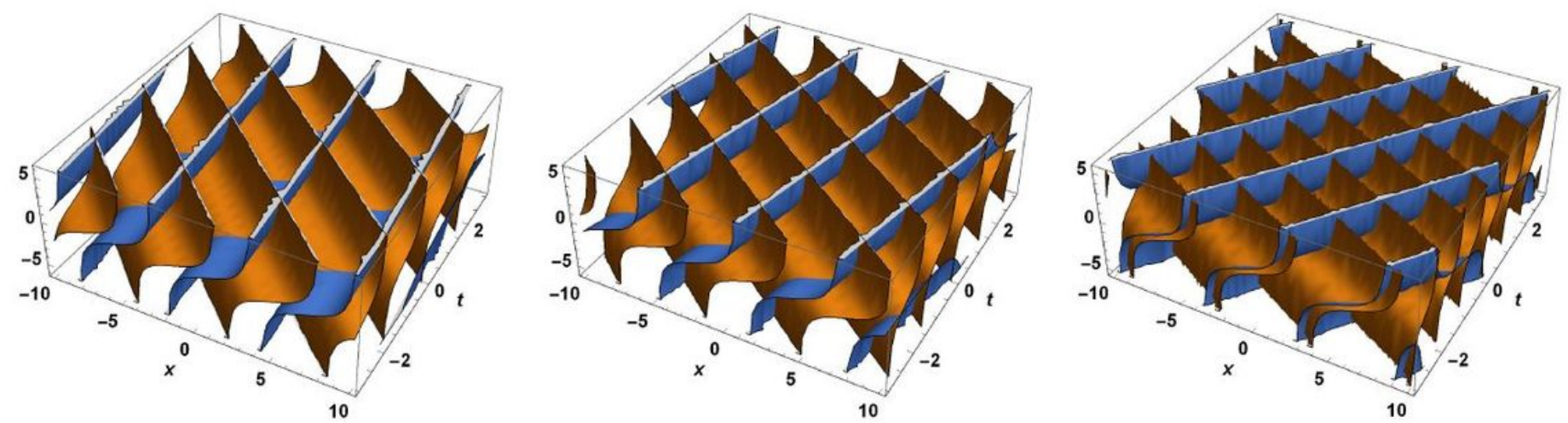
Figure 3

The interaction of the two periodic solutions upon increasing the phase velocity $s=1 ; 3 ; 5$ to the TMCH. a $=\beta=\gamma=1$. Polynomial-function method.
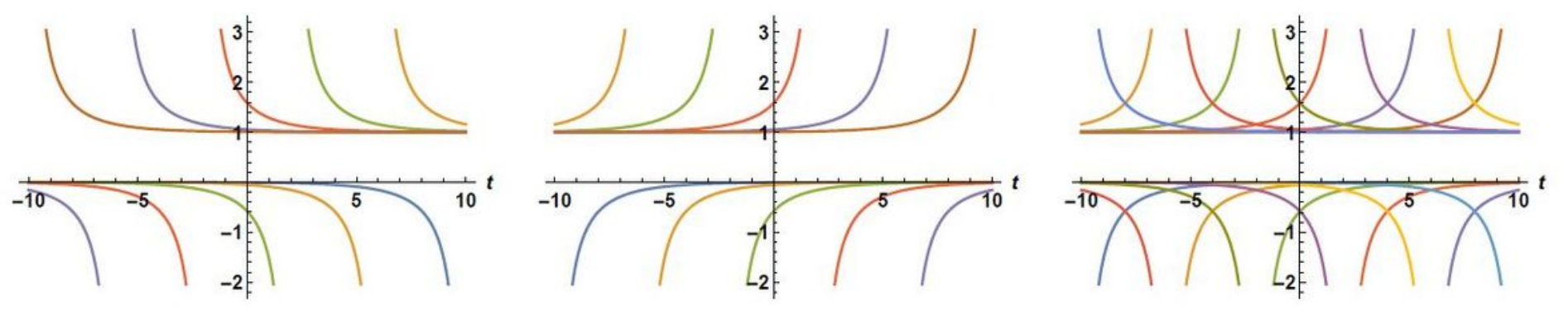

\section{Figure 4}

Prole solutions of the left, right and left-right waves to the TMBH as depicted in (4.26). $a=\beta=1 ; s=0: 5 ; b$ $=0: 5 ; d=1$.
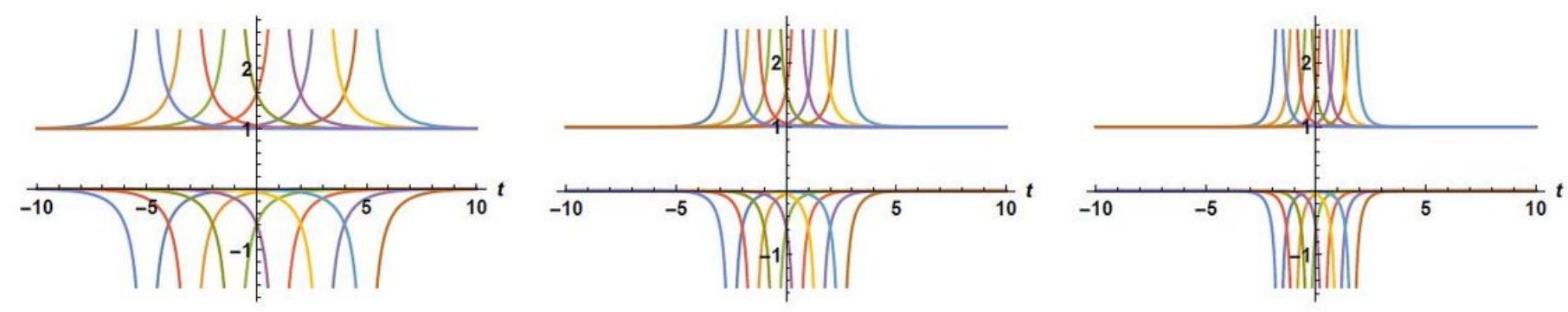

Figure 5

$2 D$ interaction of moving left-right waves upon increasing the phase velocity $s=1 ; 2 ; 3 . a=\beta=1 ; b=0: 5$; $\mathrm{d}=1$. Kudryashov-expansion method. 